\title{
Nonbacterial Thrombotic Endocarditis Associated With Systemic Lupus Erythematosus
}

\author{
Kazuaki Tanabe, MD*, Minako Kinoshita, MD*, Tomoko Tani, MD*, \\ Toshikazu Yagi, RDCS*, Masahiko Shinkai, MD**, Yukikatsu Okada, MD*, \\ Shigefumi Morioka, MD*and Yasuki Kihara, MD* \\ *Division of Cardiology \\ ** Cardiovascular Surgery, Kobe General Hospital, Kobe, Japan
}

In this report we describe the case of a 34-year-old woman who had mitral valve nonbacterial vegetation and right atrial thrombus attached to the atrial septum.

The patient was admitted to Kobe General Hospital with a 1-month history of fever. She had no history of heart disease. Physical examination revealed bilateral leg edema and apical pan-systolic murmur (grade 3). Her heart rate was 90 beats/min (sinus rhythm) and blood pressure was 110/70 $\mathrm{mmHg}$. Transthoracic and transesophageal echocardiography demonstrated attachment of a nonmobile soft vegetation to the posterior leaflet of the mitral valve (Fig. 1A) and severe mitral regurgitation due to mitral valve prolapse (Fig. 1B). The vegetation size was $10 \mathrm{~mm}$. A highly mobile right atrial mass attached to the atrial septum, mimicking a myxoma, was also visible (Fig. 2). The size of mass was 14 x $15 \mathrm{~mm}$. Her left ventricular (LV) enddiastolic dimension was $45 \mathrm{~mm}$, LV end-systolic dimension was $25 \mathrm{~mm}$, and LV ejection fraction was $68 \%$. Left atrial dimension was $37 \mathrm{~mm}$. Estimated right ventricular systolic pressure was $61 \mathrm{mmHg}$. The patient was investigated for infective endocarditis, however this work up was negative. The diagnoses of systemic lupus erythematosus (SLE), antiphospholipid antibodies syndrome, and nephrotic syndrome were con-

Received November 9, 2006; revision received January 17, 2007; accepted February 8, 2007

Address for correspondence: Kazuaki Tanabe, MD, PhD

Division of Cardiology, Kobe General Hospital,

4-6 Minatojima-nakamachi, Cyuo-ku,

Kobe 650-0046, Japan.

Telephone: +81-78-302-4321

Fax: +81-78-302-2487

E-mail: ktanabe@leto.eonet.ne.jp

(C) 2007 Japanese Society of Echocardiography

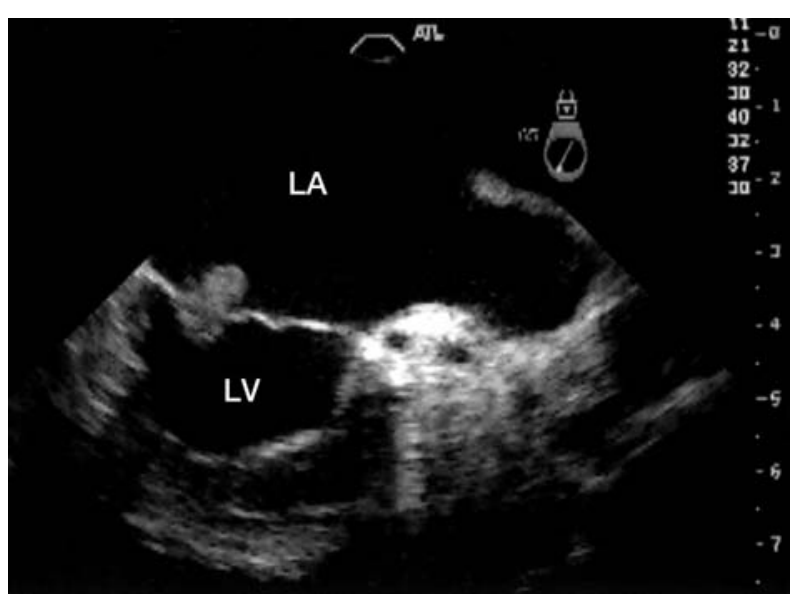

Fig $1 \mathrm{~A}$

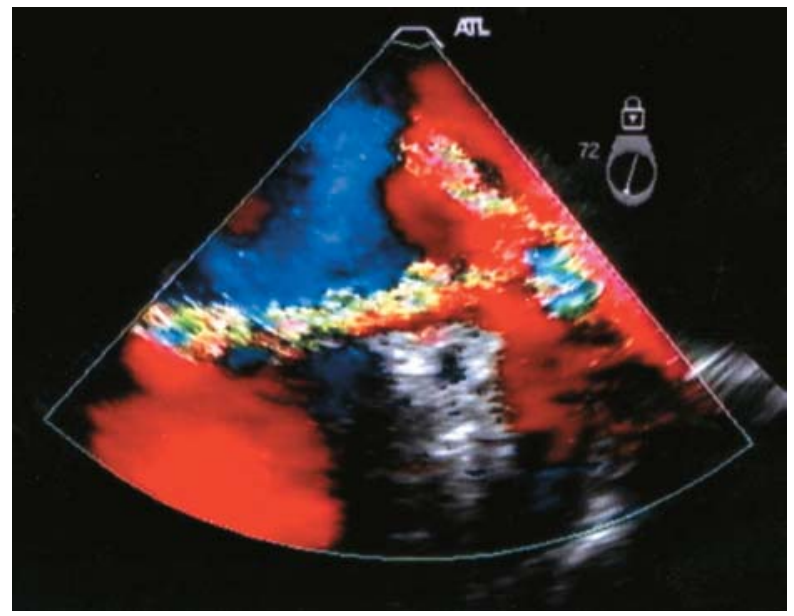

Fig 1B

Fig. 1. Transesophageal echocardiogram demonstrating a vegetation attached to the postero-medial scallop of mitral valve $(A)$ and eccentric mitral regurgitant jet (B). $L A=$ left atrium, $L V=$ left ventricle. 


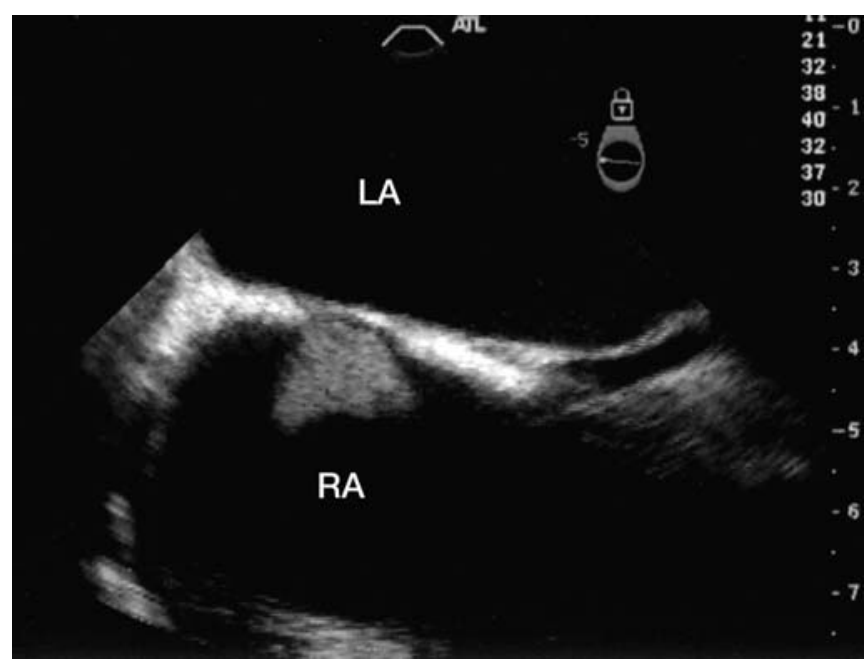

Fig. 2. Transesophageal echocardiogram demonstrating a right atrial mass attached to the fossa ovalis of the atrial septum, mimicking a myxoma. LA=left atrium, $\mathrm{RA}=$ right atrium

firmed by laboratory data.

Open-heart surgery was performed because of severe mitral regurgitation and concern about the risk of embolism. The right atrial mass attached to the fossa ovalis of the atrial septum was removed, and mitral valve plasty was then performed. The right atrial mass proved to be the thrombus and the mitral valve mass was the sterile vegetation by histological examination.

Valvular involvement in SLE is common, and valvular abnormalities have been shown by transesophageal echocardiography in more than $50 \%$ of the patients with SLE [1]. Valvular thickening is the most common echo finding, followed by vegetations and valvular regurgitation. Valvular diseases have been demonstrated to be a source of embolic cerebrovascular disease [2]. The antiphospholipid antibodies syndrome is a well-defined clinical and serological entity characterized by venous and arterial thrombosis. Clinically sig- nificant valvular or intracardiac masses may be treated by high-dose anticoagulation with warfarin [3]. However, there are no long-term controlled studies of the effect of chronic anticoagulation and valve disease.

This case offers the opportunity to emphasize the difficulty of diagnostic differentiation of intracardiac masses using echocardiographic imaging.

\section{Reference}

1. Roldan CA, Shively BK, Crawford. An echocardiographic study of valvular disease associated with systemic lipus erythematosus. N Engl J Med 1996; 335: 1424-1430

2. Roldan CA, Gelgand EA, Qualls CR, Sibbitt WL Jr.. Valvular heart disease as a cause of cerebrovascular disease in patients with systemic lupus erythematosus. Am J Cardiol 2005; 95: 1441-1447.

3. Agirbasli MA, Hansen DE, Byrde BF. Resolution of vegetations with anticoagulation after myocardial infarction in primary antiphospholipid syndrome. J Am Soc Echocardiogr 1997; 10; 877-880 